\title{
Identified particle production in inelastic $p p$ events with the ATLAS detector
}

\author{
Leonid Gladilin*i \\ Moscow State University - Scobeltsyn Institute of Nuclear Physics \\ 1(2), Leninskie gory, GSP-1, Moscow 119991 - Russia \\ E-mail: Leonid.Gladilin@cern.ch
}

\begin{abstract}
Various strange and charmed hadrons were reconstructed with the ATLAS detector in $p p$ collisions at $\sqrt{s}=7 \mathrm{TeV}$. The data sample was collected in March-May of 2010 using a minimum-bias trigger. The $K_{S}^{0}$ and $\Lambda^{0}$ kinematic distributions were studied using data corresponding to an integrated luminosity of $190 \mu \mathrm{b}^{-1}$. The $\Xi^{\mp}$ and $\Omega^{\mp}$ baryons were reconstructed in their cascade decays in data corresponding to an integrated luminosity of $250 \mu \mathrm{b}^{-1}$. The $D^{* \pm}, D^{ \pm}$and $D_{s}^{ \pm}$ charmed mesons were reconstructed in the range of transverse momentum $p_{\mathrm{T}}\left(D^{(*)}\right)>3.5 \mathrm{GeV}$ and pseudorapidity $\left|\eta\left(D^{(*)}\right)\right|<2.1$ in data corresponding to an integrated luminosity of $1.4 \mathrm{nb}^{-1}$. The fitted mass values were found to be in agreement with their world averages while the observed invariant mass resolutions agree with Monte Carlo expectations. This study confirms the high performance of the ATLAS detector for precision tracking measurements.
\end{abstract}

35th International Conference of High Energy Physics - ICHEP2010,

July 22-28, 2010

Paris France

\footnotetext{
*Speaker.

${ }^{\dagger}$ On behalf of the ATLAS Collaboration.
} 


\section{Introduction}

The reconstruction of identified particles containing strange and charm quarks is already feasible with first LHC data due to the large expected cross sections and clean particle signatures. A study of $\phi(1020) \rightarrow K^{+} K^{-}$decays with the ATLAS detector [1] in $\sqrt{s}=900 \mathrm{GeV}$ collision data has been reported earlier [2,3]. The $d E / d x$ particle identification was used to select charged kaons in that analysis. Results on identified strange and charmed particles production in $p p$ collisions at $\sqrt{s}=7 \mathrm{TeV}$ are presented in this note.

The data used in this analysis were collected between March and May, 2010. Events selected with a minimum-bias trigger and with a reconstructed primary vertex were kept for particle reconstruction. The $d E / d x$ particle identification was not used. Non-diffractive minimum-bias Monte Carlo (MC) was used to tune the selection criteria and to make comparisons with data.

\section{Strange Particle Production}

The decays $K_{S}^{0} \rightarrow \pi^{+} \pi^{-}, \Lambda^{0} \rightarrow p \pi^{-}$and $\bar{\Lambda}^{0} \rightarrow \bar{p} \pi^{+}$were reconstructed by fitting pairs of tracks to a secondary vertex in data corresponding to an integrated luminosity of $190 \mu \mathrm{b}^{-1}$ [4]. Only tracks with transverse momentum $p_{\mathrm{T}}>100 \mathrm{MeV}$ were used. The transverse distance between the secondary and primary vertices was required to be at least $4 \mathrm{~mm}$ for $K_{S}^{0}$ candidates, while the minimal there-dimensional distance of $30 \mathrm{~mm}$ was required for $\Lambda^{0}$ and $\bar{\Lambda}^{0}$ candidates. The distributions of the invariant mass of the $K_{S}^{0}$ and $\Lambda^{0}$ candidates in data and MC simulation are shown in Fig. 1. The signal positions and widths are consistent with the MC expectations and with the world average mass values [5]. Distributions of pseudorapidity and azimuthal angle for the reconstructed $K_{S}^{0}, \Lambda^{0}$ and $\bar{\Lambda}^{0}$ candidates are described reasonably well by the MC simulation, while the MC has a greater fraction of candidates at higher values of the candidate transverse momentum [4].

The cascade decays $\Xi^{-} \rightarrow \Lambda^{0} \pi^{-}$and $\Omega^{-} \rightarrow \Lambda^{0} K^{-}$with $\Lambda^{0} \rightarrow p \pi^{-}$(+ c.c.) were reconstructed by fitting secondary and tertiary vertices in data corresponding to an integrated luminosity
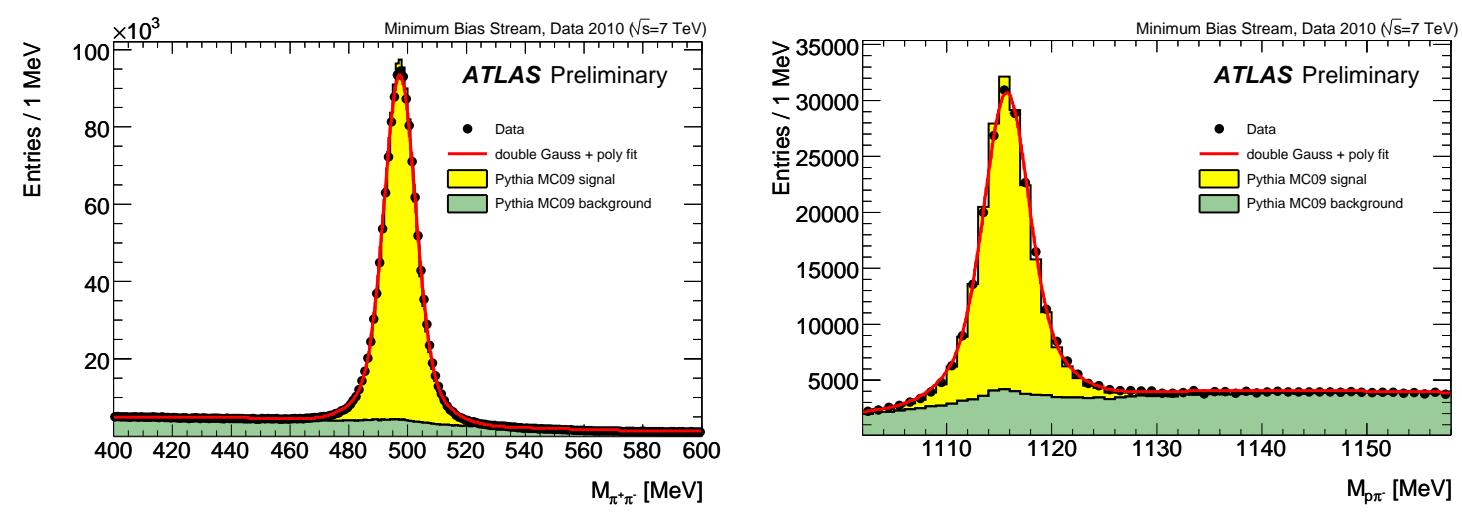

Figure 1: The $M\left(\pi^{+} \pi^{-}\right)$distribution for $K_{S}^{0}$ candidates (left plot) and $M\left(p \pi^{-}\right)$for $\Lambda^{0}$ candidates (right plot). The points are data, while the histograms show the MC simulation. The solid curves represent fit results. The fitted masses (widths) are $497.427 \pm 0.006 \mathrm{MeV}(5.60 \mathrm{MeV})$ and $1115.73 \pm 0.01 \mathrm{MeV}(2.28 \mathrm{MeV})$ for the $K_{S}^{0}$ and $\Lambda^{0}$ signals, respectively. The fit uncertainties on the mass values are statistical only. 

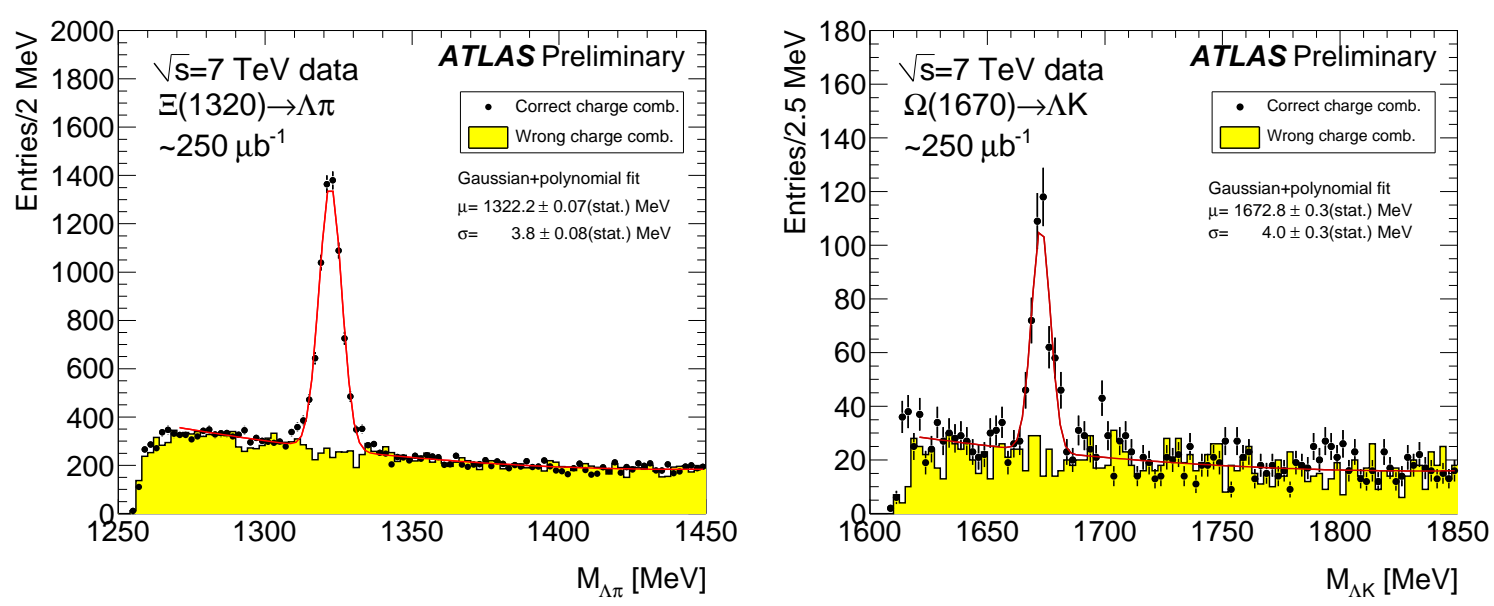

Figure 2: The $M(\Lambda \pi)$ distribution for $\Xi^{\mp}$ candidates (left plot) and $M(\Lambda K)$ for $\Omega^{\mp}$ candidates (right plot). The histograms show the distributions for wrong-charge combinations. The solid curves represent fit results.

of $250 \mu \mathrm{b}^{-1}$ [6]. The additional track for the first cascade vertex was required to have transverse momentum above $150 \mathrm{MeV}$ for $\Xi^{\mp}$ candidates and above $400 \mathrm{MeV}$ for $\Omega^{\mp}$ candidates. The transverse distance between the first cascade vertex and primary vertex was required to be at least $4 \mathrm{~mm}$ for the $\Xi^{\mp}$ candidates and at least $6 \mathrm{~mm}$ for the $\Omega^{\mp}$ candidates. The distributions of the invariant mass of the $\Xi^{\mp}$ and $\Omega^{\mp}$ candidates in data and MC simulation are shown in Fig. 2. The signal positions and widths are consistent with the MC expectations and with the world average mass values [5].

\section{Charmed Particle Production}

The $D^{* \pm}, D^{ \pm}$and $D_{s}^{ \pm}$charmed mesons were reconstructed in the range of transverse momentum $p_{\mathrm{T}}\left(D^{(*)}\right)>3.5 \mathrm{GeV}$ and pseudorapidity $\left|\eta\left(D^{(*)}\right)\right|<2.1$ in data corresponding to an integrated luminosity of $1.4 \mathrm{nb}^{-1}$ [7].

The $D^{* \pm}$ mesons were identified using the decay channel $D^{+} \rightarrow D^{0} \pi_{s}^{+} \rightarrow\left(K^{-} \pi^{+}\right) \pi_{s}^{+}$, where the pion from the $D^{*+}$ decay is referred to as the "soft" pion, $\pi_{s}$. The transverse decay length of the $D^{0}$ candidates was required to satisfy $L_{\mathrm{XY}}>0$. The left plot in Fig. 3 shows the mass difference, $\Delta M=M\left(K \pi \pi_{s}\right)-M(K \pi)$, distribution for the $D^{* \pm}$ candidates which satisfy $1.83<M(K \pi)<$ $1.90 \mathrm{GeV}$, while the right plot in Fig. 3 shows the $M(K \pi)$ distribution for the $D^{* \pm}$ candidates which satisfy $144<\Delta M<147 \mathrm{MeV}$. The fitted $D^{* \pm}$ yield was $N\left(D^{* \pm}\right)=2020 \pm 120$.

The $D^{ \pm}$mesons were reconstructed from the decay $D^{+} \rightarrow K^{-} \pi^{+} \pi^{+}$, and $D_{s}^{ \pm}$mesons were reconstructed from the decay $D_{s}^{+} \rightarrow \phi \pi^{+}$with $\phi \rightarrow K^{+} K^{-}$. The transverse decay lengths of the $D^{ \pm}$and $D_{s}^{ \pm}$candidates were required to be above $1.3 \mathrm{~mm}$ and $0.4 \mathrm{~mm}$, respectively. For $D_{s}^{ \pm}$candidates, the $M(K K)$ invariant mass was required to be within $\pm 6 \mathrm{MeV}$ of the $\phi$ mass. The $M(K \pi \pi)$ distribution for the $D^{ \pm}$candidates and the $M(K K \pi)$ distribution for the $D_{s}^{ \pm}$candidates are shown in Fig. 4. The fitted yields were $N\left(D^{ \pm}\right)=1667 \pm 86$ and $N\left(D_{s}^{ \pm}\right)=326 \pm 57$.

The fitted masses of the reconstructed charmed mesons were found to be in agreement with their world averages [5] while the observed invariant mass resolutions agree with MC expectations. 

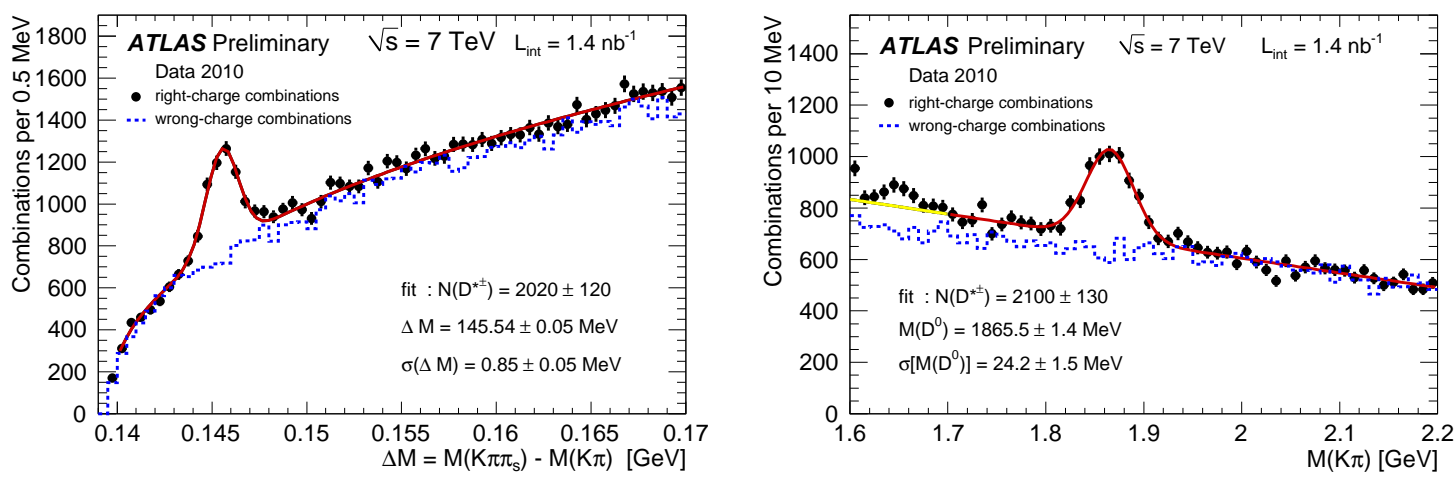

Figure 3: The distribution of the mass difference, $\Delta M=M\left(K \pi \pi_{s}\right)-M(K \pi)$, (left plot) and the $M(K \pi)$ distribution (right plot) for the $D^{* \pm}$ candidates (points). The dashed histograms show the distributions for wrong-charge combinations. The solid curves represent fit results.
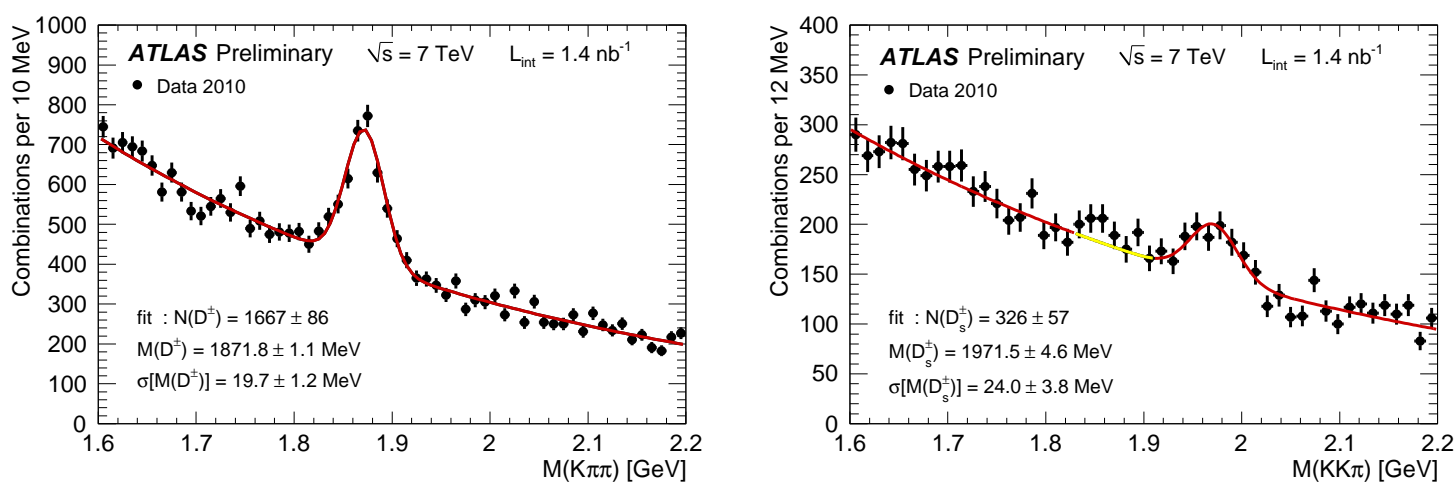

Figure 4: The $M(K \pi \pi)$ distribution for the $D^{ \pm}$candidates (left plot) and the $M(K K \pi)$ distribution for the $D_{s}^{ \pm}$candidates (right plot). The solid curves represent fit results.

\section{References}

[1] The ATLAS Collaboration, The ATLAS experiment at the CERN Large Hadron Collider, JINST 3 (2008) S08003.

[2] The ATLAS Collaboration, $\phi(1020)$-meson production in $\sqrt{s}=900 \mathrm{GeV}$ collision data, ATLAS-CONF-2010-023, http: / / cdsweb. cern. ch/record/1277655.

[3] The ATLAS Collaboration, Performance of the ATLAS Detector using First Collision Data, JHEP 09 (2010) 056.

[4] The ATLAS Collaboration, Kinematic Distributions of $K_{S}^{0}$ and $\Lambda$ decays in collision data at $\sqrt{s}=7 \mathrm{TeV}$, ATLAS-CONF-2010-033, http: / / cdsweb. cern. ch/record/1277668.

[5] K. Nakamura et al., (Particle Data Group), The Review of Particle Physics, J. Phys. G37 (2010) 075021.

[6] The ATLAS Collaboration, Observation of $\Xi, \Omega$ baryons and $K^{*}(890)$ meson production at $\sqrt{s}=7 \mathrm{TeV}$, ATLAS-CONF-2010-032, http: / / cdsweb.cern. ch/record/1277666.

[7] The ATLAS Collaboration, $D^{(*)}$ mesons reconstruction in pp collisions at $\sqrt{s}=7 \mathrm{TeV}$, ATLAS-CONF-2010-034, http: / / cdsweb. cern. ch/record/1277669. 